\title{
Velocimetry of cold atoms by matterwave interferometry
}

\author{
Max Carey, ${ }^{*}$ Jack Saywell, David Elcock, Mohammad Belal, and Tim Freegarde \\ School of Physics $\&$ Astronomy, University of Southampton, \\ Highfield, Southampton SO17 1BJ, United Kingdom
}

(Dated: February 28, 2019)

\begin{abstract}
We present an elegant application of matterwave interferometry to the velocimetry of cold atoms whereby, in analogy to Fourier transform spectroscopy, the 1-D velocity distribution is manifest in the frequency domain of the interferometer output. By using stimulated Raman transitions between hyperfine ground states to perform a three-pulse interferometer sequence, we have measured the velocity distributions of clouds of freely-expanding ${ }^{85} \mathrm{Rb}$ atoms with temperatures of $34 \mu \mathrm{K}$ and $18 \mu \mathrm{K}$. Quadrature measurement of the interferometer output as a function of the temporal asymmetry yields velocity distributions with excellent fidelity. Our technique, which is particularly suited to ultracold samples, compares favourably with conventional Doppler and time-of-flight techniques, and reveals artefacts in standard Raman Doppler methods. The technique is related to, and provides a conceptual foundation of, interferometric matterwave accelerometry, gravimetry and rotation sensing.
\end{abstract}

\section{INTRODUCTION}

In the macroscopic world, we measure an object's temperature by bringing it into thermal equilibrium with a small probe. When the object is a cloud of cold atoms, the tiny thermal mass requires a thermodynamic probe on the atomic scale [1]; and inhomogeneous cooling, incomplete thermalization, coupling to electronic energy levels, and non-equilibrium quantum thermodynamics can mean that the temperature is poorly defined [2]. It is therefore common to characterize a cold atom sample by its velocity distribution, from which the kinetic temperature may be found by subsequent parameterization.

Popular methods of measuring velocity distributions, such as time-of-flight imaging [3], Raman [4, 5] and Bragg $[6,7]$ Doppler spectroscopy, rely upon separate interactions with small slices of the velocity distribution to build up a complete measurement. Each involves the signal from only a small number of atoms, and there are artefacts from the initial cloud size and measurement-induced perturbation of the velocity distribution. Despite a variety of enhancements [8-10], precise interpretation of the results requires care $[5,11]$.

Here we present an alternative method of cold atom velocimetry by matterwave interferometry, in which broadband interactions with laser pulses allow all the atoms within the cloud to be interrogated simultaneously, permitting a significant improvement in the measurement signal-to-noise ratio while subjecting the sample to only the smallest perturbations and constraints. In contrast to Doppler spectroscopy, the technique is particularly suited to the lowest temperatures. While it can be considered a method of measuring the temperature-dependent coherence length of the atomic wavepacket [12-14], we show here that it allows the velocity distribution to be measured in detail. It provides an elegant conceptual underpinning of matterwave accelerometry, gravimetry and

*max.carey@soton.ac.uk rotation sensing, in which differential velocity measurements are performed by two sequences in succession [1519], and as a result inspires adaptations that for example offer immunity to mechanically-induced laser phase noise between the two measurements [20].

\section{CONCEPTUAL OVERVIEW}

Matterwave interferometry is performed by sequences of pulsed interactions, resonant with the two-state quantum system $(|1\rangle,|2\rangle)$, that are separated by periods of free evolution [21]. Prior to each sequence, the atoms are pumped into one of the two states, $|1\rangle$. The first pulse then places the atom into a quantum superposition of the two states, whose phase is determined by that of the

(a)

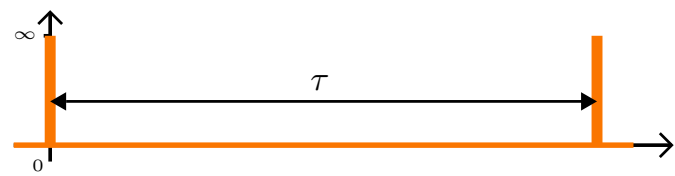

(b)

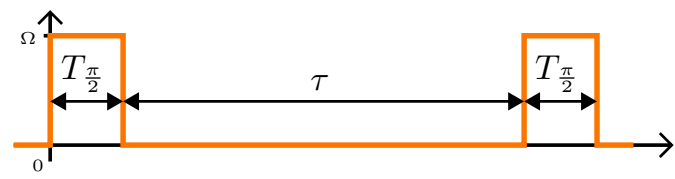

(c)

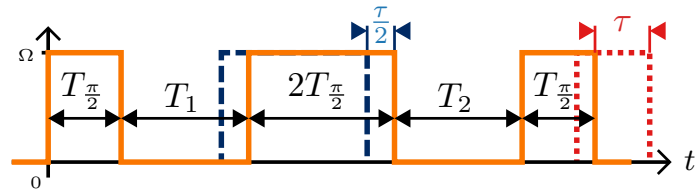

FIG. 1. Temporal pulse profiles for the interferometer sequences considered here. a) Ramsey sequence with ideal, zero-length, pulses. b) Ramsey sequence with realistic, finitelength, pulses. c) Asymmetric Mach-Zehnder sequence with realistic pulses. 


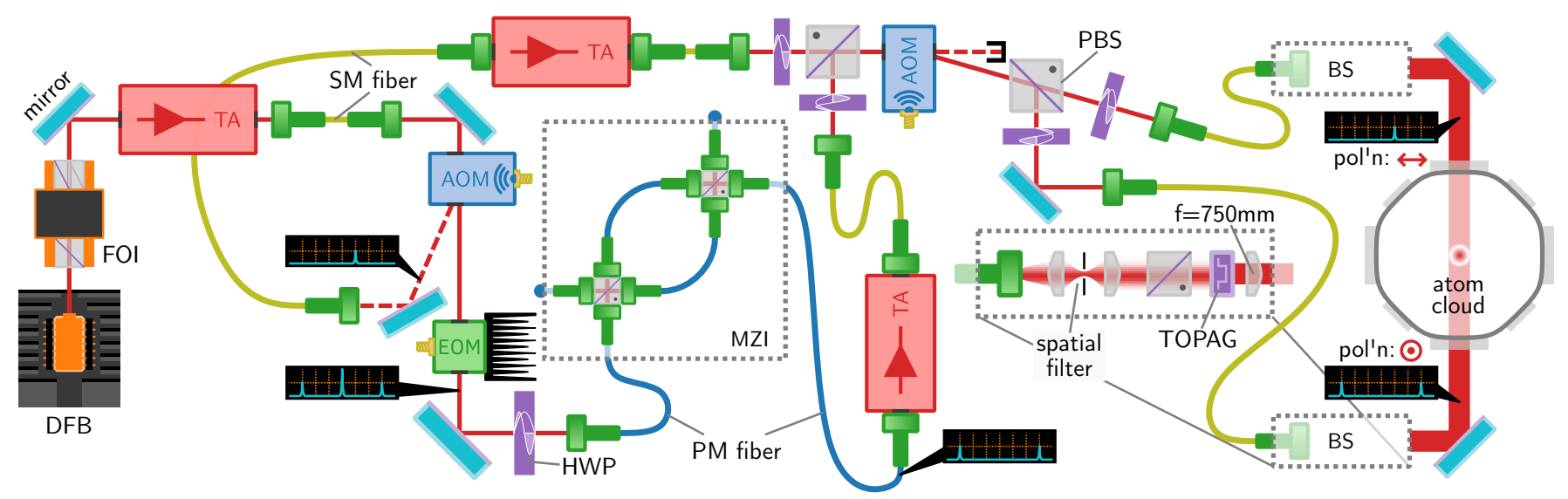

FIG. 2. Schematic for Raman interferometer: Distributed feedback laser diode (DFB), Faraday optical isolator (FOI), tapered amplifier (TA), acousto-optic modulator (AOM), electro-optic modulator (EOM), half waveplate (HWP), polarizing beam-splitter (PBS), Topag GTH-4-2.2 refractive beam-shaper (TOPAG), beam-shaping optics (BS), single-mode (SM) and polarization-maintaining (PM) fibers, fiber Mach-Zehnder filter (MZI). MOT optics are not shown, and only one instance is labeled for some repeated symbols.

interaction field. The effect of subsequent pulses then depends upon the phase difference accrued between the timebase of the interaction field and the atomic state [17].

An example is the Ramsey interferometer [22], consisting of two pulses separated by a free-evolution period $\tau$ as depicted in Figure 1a. Each " $\pi / 2$-pulse" lasts a quarter of the Rabi oscillation period, and thus converts either of the two atomic states into an equal superposition. In the case that $\tau=0$, the two pulses combine to form a " $\pi$-pulse", which transfers atoms from one state to the other.

For $\tau>0$, the first pulse leaves each atom in a superposition which then evolves freely to accrue a relative phase $\Phi$ according to the atoms' environment and trajectory. The phase determines the effect of the second pulse of the sequence, and can thus be measured by monitoring the atomic state populations once the sequence is complete.

If the interaction is with a pulsed laser beam, then atoms propagating with velocity $v_{z}$ along the beam axis will experience a Doppler-shifted field which gives the superposition phase $\Phi$ a dependence on the distance traveled during the evolution time $\tau$. If there are no other phase contributions then

$$
\Phi=\mathbf{k} \cdot \mathbf{v} \tau=k v_{z} \tau
$$

where $k$ is the laser wavenumber [23, 24]. The second pulse maps this phase onto atomic state probabilities so that, with ideal pulses that perform this mapping exactly, the probability that a given atom is in the second atomic state $|2\rangle$ is

$$
\left|c_{2}\right|^{2}=\frac{1}{2}\left[1+\cos \left(k v_{z} \tau\right)\right],
$$

and thus the fraction of a statistical ensemble in state $|2\rangle$ will be

$$
\mathcal{S}(\tau)=\int_{-\infty}^{\infty} P\left(v_{z}\right) \frac{1}{2}\left[1+\cos \left(k v_{z} \tau\right)\right] d v_{z}
$$

where $P\left(v_{z}\right)$ is the normalized distribution of velocity components $v_{z}$ in the beam direction. Each velocity class $v_{z}$ hence contributes to the interferometer signal a component which varies sinusoidally with $\tau$, with frequency $k v_{z}$ and amplitude proportional to $P\left(v_{z}\right)$, akin to the contributions from different wavelengths of light to the signal produced when varying the arm length of a Michelson interferometer in Fourier transform spectroscopy [25, 26]. The velocity distribution is thus mapped onto the frequency domain of the signal but, owing to the symmetry of the cosine function, positive and negative velocities cannot be distinguished.

If the laser phase is advanced by $\phi$ between the two interferometer pulses, this phase is mapped onto the output signal such that

$$
\mathcal{S}(\phi, \tau)=\int_{-\infty}^{\infty} P\left(v_{z}\right) \frac{1}{2}\left[1+\cos \left(k v_{z} \tau-\phi\right)\right] d v_{z} .
$$

The absolute value of the Fourier transform of the quantity

$$
\mathcal{S}_{I}+i \mathcal{S}_{Q} \equiv \mathcal{S}(0, \tau)+i \mathcal{S}(\pi / 2, \tau)
$$

is then proportional to the velocity distribution $P\left(k v_{z}\right)$, expressed as a function of the frequency $k v_{z}$ $[27,28]$. 


\section{A MACH-ZEHNDER INTERFEROMETER FOR ATOM VELOCIMETRY}

The astute reader will notice that the Fourier transform of the signal defined in Equation (5) can only be measured experimentally for positive pulse separations $\tau$, and that the signal is thus effectively multiplied by a Heaviside step function. With ideal interferometer pulses that perform perfect, instantaneous operations upon all atoms (Figure 1a) this would introduce an orthogonal component to the Fourier transform that could be separated from the velocity information, but in practice deconvolution becomes intractable because pulses of finite duration (Figure 1b) themselves exhibit Doppler sensitivity, introducing a velocity-dependent amplitude and phase shift that we have explored in more detail in [27].

In this work we therefore interleave a "mirror" $\pi$ pulse between the "beam-splitters" of our interferometer, as shown in Figure 1c. In its time-symmetrical form $\left(T_{2}=T_{1}\right)$, atoms divide their time equally between the two interferometer states, and the interferometer forms a basic "composite pulse" [29] in which systematic contributions to the phase accrued during the first evolution period $T_{1}$ are reversed during the second pe$\operatorname{riod} T_{2}$. This Mach-Zehnder arrangement forms the basis for atom interferometric inertial sensing, since steady velocity-dependent phase shifts cancel and only the phase shifts due to changes in velocity remain [15-19].

When the interferometer is asymmetric, however, we retain the velocity sensitivity according to the temporal asymmetry $\tau=T_{2}-T_{1}$, which can be varied continuously over both negative and positive values, while taking advantage of partial cancellation of phase shifts accrued during the pulses themselves [30].

To maintain a constant atom cloud expansion in our experiments, we set the total interferometer duration $T=T_{1}+T_{2}$ to a constant and vary $\tau$ between $-T$ and $T$. We show in Appendix A that some Doppler sensitivity remains in a velocity-dependent modification of the fringe amplitude that could be corrected for in subsequent analysis, and the introduction of sub-harmonics with twice the fringe period that enhance the apparent probability of lower atomic velocities. Provided the Doppler shift is no more that 0.4 of the Rabi frequency, however, these effects are negligible.

\section{EXPERIMENTAL PROCEDURE}

Our experimental apparatus [29, 31] is shown schematically in Figure 2. A 3D magneto-optical trap (MOT) of ${ }^{85} \mathrm{Rb}$ atoms is formed using a cooling laser detuned by the order of the natural linewidth to the red of the $\left|5 S_{1 / 2}, F=3\right\rangle \rightarrow\left|5 P_{3 / 2}, F^{\prime}=4\right\rangle$ cycling transition, and a repump laser locked to the $\left|5 S_{1 / 2}, F=2\right\rangle \rightarrow$ $\left|5 P_{3 / 2}, F^{\prime}=3\right\rangle$ transition ensures that atoms are not lost from the cooling cycle to the $\left|5 P_{3 / 2}, F^{\prime}=3\right\rangle$ state. After a $1 \mathrm{~s}$ loading time, the magnetic field gradient is extin-

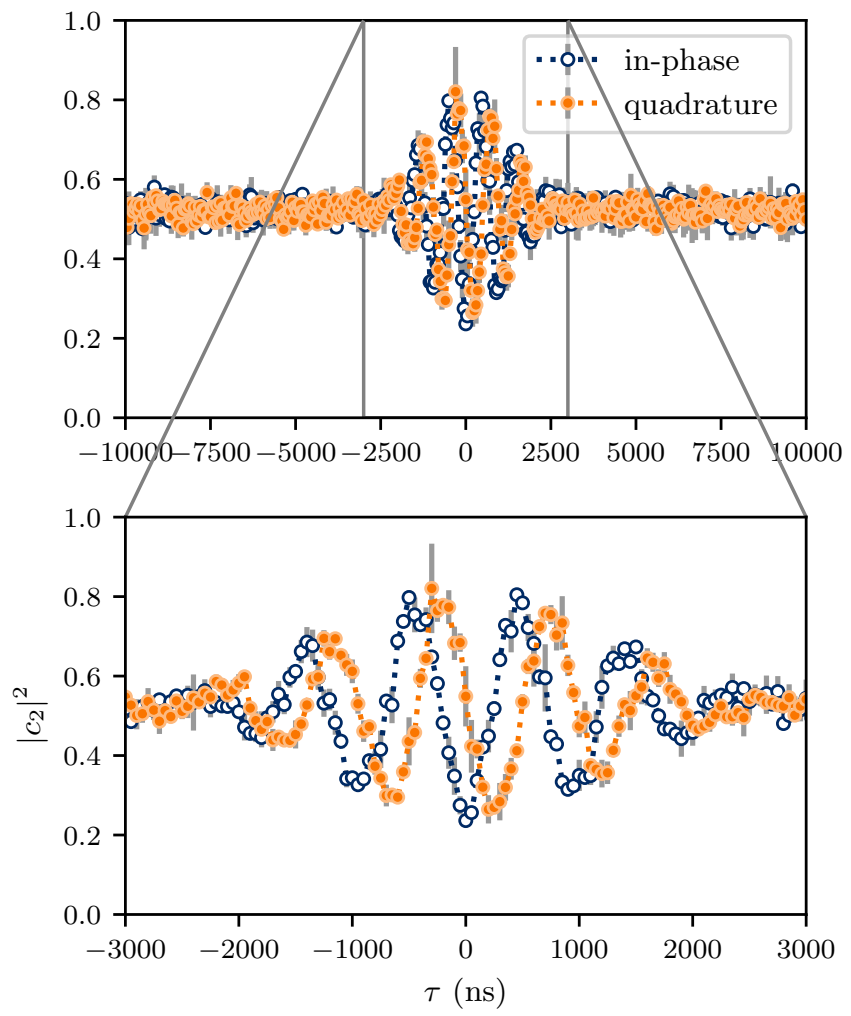

FIG. 3. Measurements of fractional population transfer $\left|c_{2}\right|^{2}$ as a function of temporal asymmetry $\tau$ for interferometers with (filled orange circles) and without (open blue circles) a $\pi / 2$ phase shift before the final recombination pulse. Each point is an average of two measurements, with gray error bars representing the standard deviation. A detuning of $\delta_{\text {laser }}=-2 \pi \times 1050 \mathrm{kHz}$ from the hyperfine splitting results in oscillations which appear within an envelope whose shape is governed by the velocity distribution.

guished and the cooling laser reduced in power, leaving the atoms to undergo sub-Doppler cooling in an optical molasses. After a further period of typically $11 \mathrm{~ms}$, the repump laser is extinguished and the cooling beam optically pumps the atoms into the $\left|5 S_{1 / 2}, F=2\right\rangle$ state for $\sim 4 \mathrm{~ms}$ with a $1 / \mathrm{e}$ time constant on the order of $100 \mu \mathrm{s}[32]$. The power levels and timescales are varied in order to achieve different sample temperatures, but the time between magnetic field extinction and the interferometry sequence is always kept to $15 \mathrm{~ms}$ in order to ensure that the magnetic field, which continues to vary slightly through eddy effects, is correctly nulled at the time of the interferometer sequence by additional shim coils such that the magnetic sublevels are degenerate to within $<2 \pi \times 50 \mathrm{kHz}$.

Our interferometry is conducted with two-photon Raman transitions between the $\left|5 S_{1 / 2}, F=2\right\rangle$ and $\left|5 S_{1 / 2}, F=3\right\rangle$ hyperfine ground states which have a frequency splitting of $\omega_{0}=2 \pi \times 3.036 \mathrm{GHz}$. Two counter-propagating laser beams, differing in frequency 


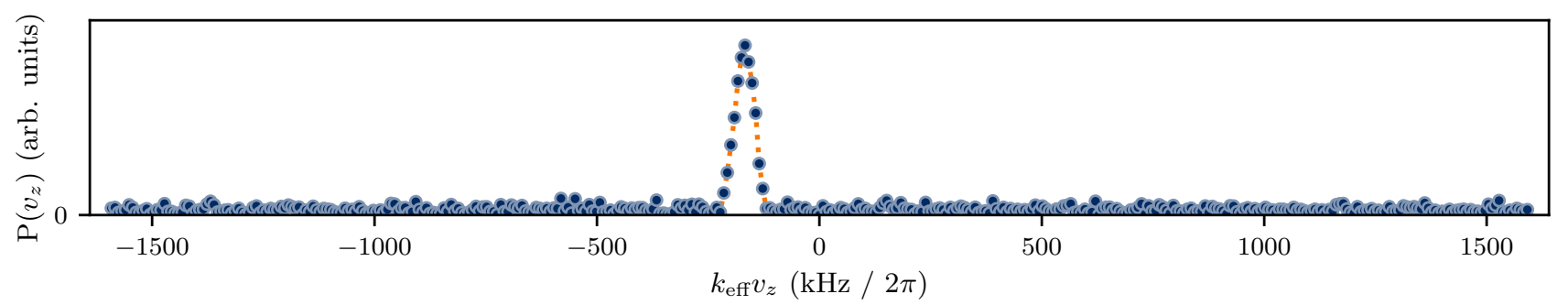

FIG. 4. Absoute value of raw FFT of data in Figure 3 after baseline subtraction, prior to subtracting the $\delta_{\text {laser }}=-2 \pi \times 1050 \mathrm{kHz}$ laser detuning from the hyperfine splitting, which manifests as a $-410 \mathrm{~mm} \mathrm{~s}^{-1}$ shift to the center of the velocity distribution. The quadrature measurement is able to resolve the sign of the displacement, so there is no component with an opposite shift.

by $\omega_{0}+\delta_{\text {laser }}$, where $\delta_{\text {laser }}$ is a variable detuning, are detuned from the $\left|5 S_{1 / 2}, F=3\right\rangle \rightarrow\left|5 P_{3 / 2}, F^{\prime}=4\right\rangle$ transition by $\Delta_{1 \text {-photon }} \approx 2 \pi \times 5 \mathrm{GHz}$. This allows long-lived ground states to be used, while the Doppler sensitivity is characterized by an effective wavenumber $k_{\text {eff }}=2 \pi / \lambda_{\text {eff }}$, with $\lambda_{\text {eff }} \approx(780 / 2) \mathrm{nm}$.

These beams are derived from an amplified freerunning distributed feedback (DFB) laser (Eagleyard EYP-DFB-0780-00080-1500-BFW01-0005), with the higher frequency beam formed by the first upper diffracted order from a $310 \mathrm{MHz}$ acousto-optic modulator $(\mathrm{AOM})$ and the lower frequency shift achieved with a $2.7 \mathrm{GHz}$ electro-optic modulator (EOM), from which the carrier frequency is suppressed by a fiber Mach-Zehnder interferometer [33]. The high frequency EOM sideband remains and, while playing no part in the Raman transition, contributes to the AC Stark shift.

The beams are separately amplified with tapered amplifier diodes, combined on a further $\mathrm{AOM}$ for fast ( $\sim 50 \mathrm{~ns})$ shuttering, and then separated again by polarization in order to be coupled into separate fibers which transfer the light to the MOT chamber. At the fiber outputs the beams are orthogonally, linearly, polarized and pass through Topag GTH-4-2.2 refractive beam shapers which give them a $1.4 \mathrm{~mm}$ square profile with $\sim 15 \%$ intensity variations across the MOT cloud at the focus of $750 \mathrm{~mm}$ focal length lenses. This allows for an intensity around $5 \mathrm{~W} \mathrm{~cm}^{-2}$ per frequency component over the interferometry region.

The interferometer read-out is performed by illuminating the atoms with the MOT cooling light for $300 \mu \mathrm{s}$ and collecting the fluorescence onto a Hamamatsu H742250 photo-multiplier tube (PMT). The atoms are then pumped back into the $\left|5 S_{1 / 2}, F=3\right\rangle$ for $\sim 50 \mu$ s by the resonant repump light [32], and then re-illuminated by the cooling light for a further $300 \mu \mathrm{s}$. Exponential decay functions are fitted to the cooling fluorescence PMT signals, and the ratio of their amplitudes is used as a measure of the fraction of atoms transferred to the $\left|5 S_{1 / 2}, F=3\right\rangle$ state during the interferometer. Full details and characterisation of the read-out process can be found in [34].

The AC Stark shift is determined by measuring the population transfer of a $\pi$-pulse measured as a function of the Raman detuning $\delta_{\text {laser }}$ before interferometry. The detuning is then set to coincide with the peak transfer. This detuning introduces a shift in the frequency domain of the signal which should be subtracted from the derived velocity distribution in order to extract a representative result. When this shift is greater than the width of the Doppler profile, the DC component of the signal does not coincide with any atomic velocity so that there is no ambiguity in analysis.

\section{RESULTS}

Figure 3 shows typical output from the interferometer, with a detuning $\delta_{\text {laser }}=-2 \pi \times 1050 \mathrm{kHz}$ introduced from the two-photon Raman resonance to offset the AC Stark shift during the pulses. The in-phase and quadrature fringes correspond to $-\mathcal{S}_{I}$ and $\mathcal{S}_{Q}$ from Equation (5), with the inversion of the in-phase component arising from the additional rotation by $\pi$. The fast Fourier transform (FFT) of these data gives the velocity distribution, without need for further determination of fringe visibility, and is shown, in units of velocity, in Figure 4.

The velocity resolution of the FFT is determined by the range of $\tau$, limited in principle to the time it takes atoms to leave the interaction region. Our data, in the range $|\tau|<10 \mu$ s (spanning $\Delta \tau=20 \mu \mathrm{s}$ ), give a velocity resolution of $\delta v=1 /\left(k_{\text {eff }} \Delta \tau\right) \approx 20 \mathrm{~mm} \mathrm{~s}^{-1}$. The value $\delta \tau$ by which $\tau$ is incremented between adjacent data points, in this case $\delta \tau=50 \mathrm{~ns}$, determines the range of velocities that can be measured $\Delta v=1 /\left(k_{\text {eff }} \delta t\right)$, although oversampling reduces the sensitivity of the measurement to the noise on any individual data point.

The absolute values of the FFT data [28] are reproduced with the two-photon detuning subtracted in Figure $5 \mathrm{a}$, where they are overlaid upon a measurement made by conventional Raman Doppler spectroscopy. Fitted Gaussian profiles, with temperatures of $31.0 \pm 1.5 \mu \mathrm{K}$ and $33.6 \pm 1.5 \mu \mathrm{K}$ respectively, are shown.

The FFT profile, shown in Figure 5(a), requires a small correction to account for a slight dependence of the fringe amplitude $\mathcal{A}\left(k_{\mathrm{eff}} v_{z}\right)$ upon atomic velocity. Figure 5(b) shows this velocity dependence (dotted line), together with the corrected velocity distribution which yields the 


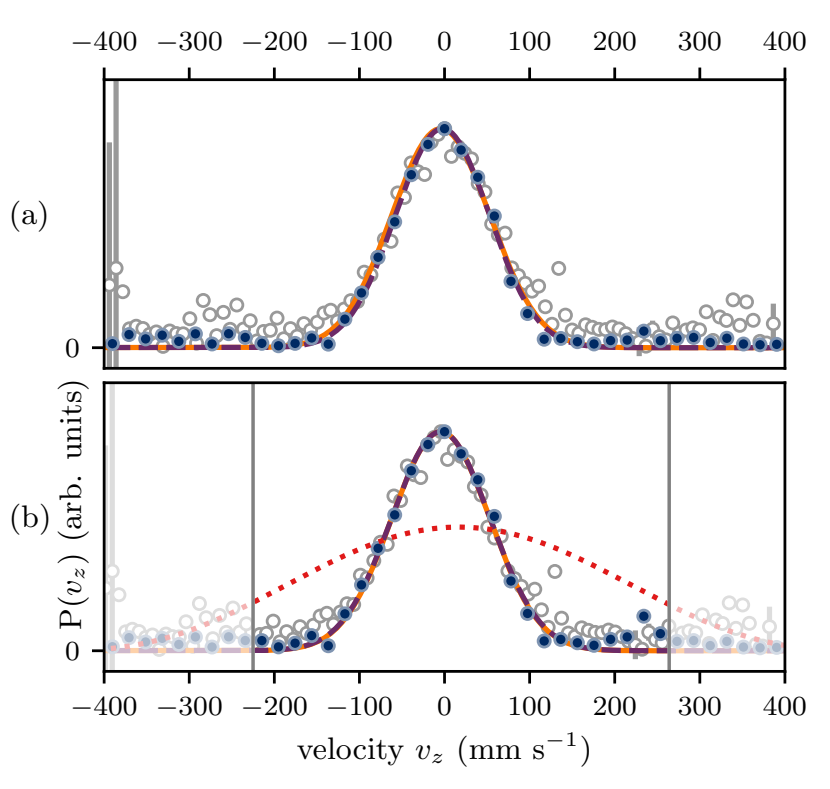

FIG. 5. Absolute value of the FFT of data in Figure 3, filled (blue) circles, overlaid upon a velocity profile determined by Raman Doppler spectroscopy, empty (gray) circles, in units of velocity. Solid (orange) lines are a Gaussian fit to the spectroscopic data, with a temperature of $33.6 \pm 1.5 \mu \mathrm{K}$, and dashed (purple) lines show Gaussian fits to the interferometric data. Plot (a) uses raw FFT data and the Gaussian fit is narrower than the spectroscopic measurement with a temperature of $31.0 \pm 1.5 \mu \mathrm{K}$. Plot (b) has a correction factor applied, multiplying each point by the reciprocal of the theoretical amplitude $\mathcal{A}\left(k_{\text {eff }} v_{z}\right)$ based on our experimental parameters, overlaid as a dashed (red) line. Only points in the $\mathcal{A}>0.4$ range (highlighted) were corrected to avoid amplifying noise at the extremities, bringing the fitted temperature to $34.1 \pm 1.6 \mu \mathrm{K}$ in agreement with the fit to the spectroscopic data. The AC Stark shift-induced offset from the spectroscopic data has been subtracted, centering it on the interferometric data at $v_{z}=-4.5 \mathrm{~mm} \mathrm{~s}^{-1}$ to better compare their shapes.

same temperature as the Doppler measurements, with notably enhanced signal-to-noise ratio. This velocity dependence, together with some parasitic contributions to the interferometer output at large detunings, are discussed in Appendix A, and impose an effective upper limit on the range of velocities that can be measured. As these effects depend on the Rabi frequency $\Omega_{R}$, higher intensity Raman beams could be used to extend this limit. The profile in this instance is slightly displaced to account for a difference of $2 \pi \times 40 \mathrm{kHz}$ between the assumed laser detuning $\delta_{\text {laser }}$ and the AC Stark shift recorded for this experiment.

The Gaussian fit to the corrected data is centered at $v_{z}=-4.5 \pm 1.3 \mathrm{~mm} \mathrm{~s}^{-1}$. This is in agreement with a second measurement, shown in Figure 7 , centered at $v_{z}=-5.1 \pm 0.7 \mathrm{~mm} \mathrm{~s}^{-1}$. We note that the two-photon recoil velocity for ${ }^{85} \mathrm{Rb}$ is $12 \mathrm{~mm} \mathrm{~s}^{-1}$; and there could be an impulse imparted to the cloud as the magnetic field is terminated.

\section{DISCUSSION}

The velocity distribution determined from our interferometric measurements agrees well for a warm sample with that obtained by conventional Doppler spectroscopy using low power Raman pulses (single-photon detuning $\sim 2 \pi \times 15 \mathrm{GHz}$, Rabi frequency $\Omega_{R} \approx 2 \pi \times 25 \mathrm{kHz}$ ). Whereas the interferometric measurements are well represented by a single Gaussian, however, the Doppler measurements show an additional, broader, component. This is particularly noticeable when a cooler sample, such as that shown in Figure 6, is probed with a strong Raman field (single-photon detuning $\sim 2 \pi \times 7 \mathrm{GHz}$, Rabi frequency $\Omega_{R} \approx 2 \pi \times 50 \mathrm{kHz}$ ), yielding a central Gaussian distribution with a temperature of $17.8 \pm 0.9 \mu \mathrm{K}$ superimposed upon a much broader background.

Interferometric measurement under the same conditions, shown in Figure 7, does not display this broad component, but modeling suggests that this is not a limitation of the interferometric technique. We have previously attributed the broad background to inhomogeneous subDoppler cooling [35]; such a distribution might also result if the Doppler technique detected warmer, untrapped atoms outside the region interrogated by the interferometer. The dependence upon the strength of the probe laser in the Doppler measurements, however, suggests that the broadening is an artefact of conventional Doppler methods, perhaps due to off-resonant excitation [5]. This is consistent with several determinations of the atom cloud temperature from measurements of the coherence length

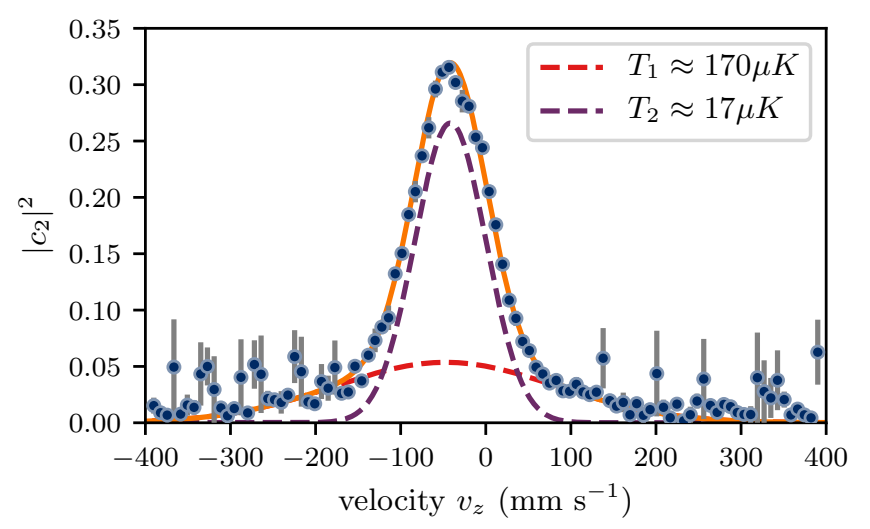

FIG. 6. Doppler spectroscopy velocity profile taken with Raman $\pi$-pulses with a single-photon detuning $\sim 2 \pi \times 7 \mathrm{GHz}$ and a Rabi frequency $\Omega_{R} \approx 2 \pi \times 50 \mathrm{kHz}$. A single Gaussian does not make a good fit, but a two Gaussian distribution fits well. The solid (orange) line is the sum of the two dashed Gaussians, a cold central peak and a broader background which we have previously attributed to inhomogenous sub-Doppler cooling. The resonance is AC Stark shifted, resulting in the large offset of the distribution from $v_{z}=0$. 
of the atomic wavepacket by measuring the fringe contrast as a function of wavepacket separation, in each case yielding a temperature below that estimated by Doppler [36] or time-of-flight [12-14] methods.

Time-of-flight measurements are often used for colder samples and condensates [37], but these are limited by the physical extent of the cloud and the imaging resolution [11]. In practice this means that the time of expansion required to measure the coldest distributions is typically $\sim 10 \mathrm{~ms}$, limiting its usefulness when studying dynamic behaviour such as in [38]. Both interferometric and Doppler measurements can be performed faster; such measurements with a Fourier transform limited resolution equivalent to Figure 7 for a $10 \mathrm{nK}$ cloud could be made in as little as $500 \mu \mathrm{s}$. However, the Doppler measurement requires a continuous interaction for this time, increasing the probability of the artefacts we have observed while limiting the resonance to a small number of atoms and reducing the signal-to-noise ratio.

Interferometric measurement, in contrast, ideally involves interactions that last for a small fraction of the total measurement time and interact uniformly with the entire velocity and spatial distribution of the atom cloud so that, on average, half of the atoms contribute to the signal, limited by the finite range that can be addressed in practice. Interferometric velocimetry is hence a particularly effective complement to existing methods and is particularly suitable for colder atom samples in which artefacts such as off-resonant excitation, saturation and scattering force heating would otherwise distort the measured velocity distributions. It uses techniques, apparatus and, in some cases [7, 12], datasets that are often already to hand.

\section{CONCLUSION}

We have described the use of Ramsey matterwave interferometry for the measurement of the velocity distribution, and hence translational temperature, of ultracold rubidium atoms. By using an asymmetrical 3-pulse arrangement with switchable pulse phases, we record quadrature signals over both positive and negative effective interferometer durations. The Fourier transform, with correction for the residual Doppler effect within the interferometer pulses themselves, then reveals the atomic velocity distributions with good fidelity because the whole atomic sample contributes to each data point. The technique can distinguish between positive and negative velocities with a quadrature measurement, and is more effective at lower cloud temperatures. It is in many ways complementary to conventional techniques of Doppler-sensitive spectroscopy and time-of-flight measurement, as it is not limited by off-resonant excitation [5] or the physical extent of the atom cloud and measurement beam [11].

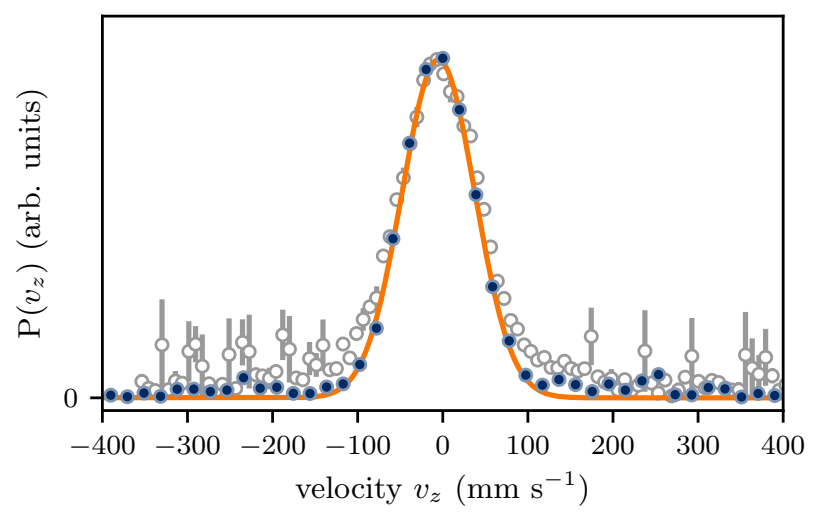

FIG. 7. Interferometric velocimetry measurement, filled circles (blue), overlaid upon the Doppler spectroscopy profile from Figure 6, empty circles (gray). The measurements were taken under the same conditions, with the offset subtracted from the spectroscopic data to center them on the interferometric profile at $v_{z}=-5.1 \mathrm{~mm} \mathrm{~s}^{-1}$. The solid (orange) line shows a Gaussian fit to the interferometric data, with a temperature of $18.7 \pm 0.6 \mu \mathrm{K}$ corresponding closely to the $17.8 \pm 0.9 \mu \mathrm{K}$ colder Gaussian fitted in Figure 6. The signalto-noise ratio of the interferometric measurement is good, and does not show signs of the broad background evident in the spectroscopic measurement.

\section{Appendix A: Analytical model for interferometer output}

Here we derive analytical expressions for the output of a Mach-Zehnder atom interferometer, with a temporal asymmetry $\tau$ between the two periods of free evolution. We consider two ways of introducing this asymmetry:

(a) keeping the total interferometer time constant such that $T_{1}=T-\tau / 2$ and $T_{2}=T+\tau / 2$, illustrated by the dashed (blue) line in Figure 1c, and

(b) keeping one separation constant such that $T_{1}=T$ and $T_{2}=T+\tau$, illustrated by the dotted (red) line in Figure 1c.

We model the effect of these sequences on a two-level atom with state amplitudes $c_{1}$ and $c_{2}$, assuming completely coherent evolution and treating pulses in the rotating wave approximation [39].

Between the pulses, the superposition accrues phase at a rate equal to the atom-laser detuning $\Delta=\delta_{\text {laser }}+$ $\delta_{\text {doppler }}$, in which we include the laser detuning from the state splitting $\delta_{\text {laser }}$ in addition to the velocity-dependent detuning $\delta_{\text {doppler }}=k v_{z}$. Free evolution for a period $T$ can then be represented by the matrix

$$
\mathbf{U}(T)=\left(\begin{array}{cc}
e^{-i \Delta \cdot T / 2} & 0 \\
0 & e^{i \Delta \cdot T / 2}
\end{array}\right)
$$

acting on a state vector $\left(\begin{array}{l}c_{1} \\ c_{2}\end{array}\right)$. 
During the pulses, the superposition undergoes a rotation in Hilbert space [40] whose rate and orientation are determined by the on-resonance Rabi frequency $\Omega$ and the detuning $\Delta_{\mathrm{ac}}=\Delta-\delta_{\mathrm{ac}}$, which differs from the inter-pulse detuning by a term $\delta_{\text {ac }}$ due to the AC Stark shift.

The effect of a pulse of duration $T$ can be solved analytically and, following the formalism of Stoner et al [41], can be represented by the matrix

$$
\boldsymbol{\Omega}\left(T, \phi_{L}\right)=\left(\begin{array}{cc}
C(T) & -i S\left(T, \phi_{L}\right) \\
-i S^{*}\left(T, \phi_{L}\right) & C^{*}(T)
\end{array}\right)
$$

to act on a state vector, with

$$
\begin{aligned}
C(T)= & \cos \left(\frac{T}{2} \sqrt{\Delta_{\mathrm{ac}}^{2}+\Omega^{2}}\right) \\
& +i \frac{\Delta_{\mathrm{ac}}}{\sqrt{\Delta_{\mathrm{ac}}^{2}+\Omega^{2}}} \sin \left(\frac{T}{2} \sqrt{\Delta_{\mathrm{ac}}^{2}+\Omega^{2}}\right), \\
S\left(T, \phi_{L}\right)= & \frac{\Omega e^{i \phi_{L}}}{\sqrt{\Delta_{\mathrm{ac}}^{2}+\Omega^{2}}} \sin \left(\frac{T}{2} \sqrt{\Delta_{\mathrm{ac}}^{2}+\Omega^{2}}\right) .
\end{aligned}
$$

Here we have included an explicit dependence on the laser phase $\phi_{L}$.

After a pure state $\left(c_{1}=1, c_{2}=0\right)$ has been subjected to an interferometer sequence, the excited state probability $\left|c_{2}\right|^{2}$ can then be calculated with matrix multiplication.

We denote the $n$th pulse with subscripts $\boldsymbol{\Omega}_{n}, C_{n}, S_{n}$ etc. so that the output of a 3 -pulse interferometer with pulse separations $T_{1,2}$ is

$$
\begin{aligned}
\left|c_{2}\right|^{2}= & \left|\left(\begin{array}{cc}
0 & 0 \\
0 & 1
\end{array}\right) \boldsymbol{\Omega}_{3} \mathbf{U}\left(T_{2}\right) \boldsymbol{\Omega}_{2} \mathbf{U}\left(T_{1}\right) \boldsymbol{\Omega}_{1}\left(\begin{array}{c}
1 \\
0
\end{array}\right)\right|^{2}, \\
= & \left|S_{1}\right|^{2}\left|S_{2}\right|^{2}\left|S_{3}\right|^{2}+\left|C_{1}\right|^{2}\left|S_{2}\right|^{2}\left|C_{3}\right|^{2} \\
& -2 \operatorname{Re}\left[e^{i \Delta\left(T_{2}-T_{1}\right)} C_{1} S_{1}\left(S_{2}^{*}\right)^{2} C_{3}^{*} S_{3}\right] \\
& +\left|S_{1}\right|^{2}\left|C_{2}\right|^{2}\left|C_{3}\right|^{2}+\left|C_{1}\right|^{2}\left|C_{2}\right|^{2}\left|S_{3}\right|^{2} \\
& +2 \operatorname{Re}\left[e^{i \Delta\left(T_{1}+T_{2}\right)} C_{1}^{*} S_{1}^{*}\left(C_{2}^{*}\right)^{2} C_{3}^{*} S_{3}\right] \\
& +2\left(\left|C_{3}\right|^{2}-\left|S_{3}\right|^{2}\right) \operatorname{Re}\left[e^{-i \Delta \cdot T_{1}} C_{1} S_{1} C_{2} S_{2}^{*}\right] \\
& +2\left(\left|C_{1}\right|^{2}-\left|S_{1}\right|^{2}\right) \operatorname{Re}\left[e^{i \Delta \cdot T_{2}} C_{2}^{*} S_{2}^{*} C_{3}^{*} S_{3}\right]
\end{aligned}
$$

To proceed we note that, for the Mach-Zehnder interferometers under consideration,

$$
\begin{aligned}
C_{3} & =C_{1}, \\
S_{3} & =e^{i \phi} S_{1}, \\
\arg \left(S_{1}\right) & =\arg \left(S_{2}\right) \\
\Rightarrow S_{1}^{*} S_{2} & =S_{1} S_{2}^{*}=\left|S_{1}\right|\left|S_{2}\right|,
\end{aligned}
$$

where $\phi$ is an advance in the laser phase introduced prior to the final pulse. This allows us to write Equation (A4) as

$$
\begin{aligned}
\left|c_{2}\right|^{2}= & \left|S_{1}\right|^{4}\left|S_{2}\right|^{2}+\left|C_{1}\right|^{4}\left|S_{2}\right|^{2}+2\left|S_{1}\right|^{2}\left|C_{2}\right|^{2}\left|C_{1}\right|^{2} \\
- & 2\left|C_{1}\right|^{2}\left|S_{1}\right|^{2}\left|S_{2}\right|^{2} \cos \left[\Delta\left(T_{2}-T_{1}\right)+\phi\right] \\
+ & 2\left|S_{1}\right|^{2} \operatorname{Re}\left[C_{1}^{2} C_{2}^{2}\right] \cos \left[\Delta\left(T_{1}+T_{2}\right)+\phi\right] \\
+ & 2\left|S_{1}\right|^{2} \operatorname{Im}\left[C_{1}^{2} C_{2}^{2}\right] \sin \left[\Delta\left(T_{1}+T_{2}\right)+\phi\right] \\
+ & 2\left(\left|C_{1}\right|^{2}-\left|S_{1}\right|^{2}\right)\left|S_{1}\right|\left|S_{2}\right| \operatorname{Re}\left[C_{1} C_{2}\right] \\
& \times\left[\cos \left(\Delta \cdot T_{1}\right)+\cos \left(\Delta \cdot T_{2}+\phi\right)\right] \\
+ & 2\left(\left|C_{1}\right|^{2}-\left|S_{1}\right|^{2}\right)\left|S_{1}\right|\left|S_{2}\right| \operatorname{Im}\left[C_{1} C_{2}\right] \\
& \times\left[\sin \left(\Delta \cdot T_{1}\right)+\sin \left(\Delta \cdot T_{2}+\phi\right)\right] .
\end{aligned}
$$

Let us first consider case (a), the interferometer keeping the free-evolution time constant, such that $T_{1,2}=$ $T \mp \tau / 2$. Noting that

$$
\begin{gathered}
\sin \left[\Delta\left(\frac{\tau}{2}+T\right)+\phi\right]+\sin \left[\Delta\left(T-\frac{\tau}{2}\right)\right]= \\
2 \sin \left(\Delta \cdot T+\frac{\phi}{2}\right) \cos \left(\Delta \frac{\tau}{2}+\frac{\phi}{2}\right), \text { and } \\
\cos \left[\Delta\left(\frac{\tau}{2}+T\right)+\phi\right]+\cos \left[\Delta\left(T-\frac{\tau}{2}\right)\right]= \\
2 \cos \left(\Delta \cdot T+\frac{\phi}{2}\right) \cos \left(\Delta \frac{\tau}{2}+\frac{\phi}{2}\right),
\end{gathered}
$$

the output can be shown to be

$$
\begin{aligned}
\left|c_{2}\right|^{2}=\frac{1}{2}\{\mathcal{C} & -\mathcal{A} \cos (\Delta \tau+\phi) \\
& \left.+\mathcal{B} \cos \left[\frac{1}{2}(\Delta \tau+\phi)\right]\right\} .
\end{aligned}
$$

with

$$
\begin{aligned}
\mathcal{A}= & 4\left|C_{1}\right|^{2}\left|S_{1}\right|^{2}\left|S_{2}\right|^{2} \\
\mathcal{B}= & 8\left(\left|C_{1}\right|^{2}-\left|S_{1}\right|^{2}\right)\left|S_{1}\right|\left|S_{2}\right| \\
& \times\left[\operatorname{Re}\left(C_{1} C_{2}\right) \cos (\Delta \cdot T+\phi / 2)\right. \\
& \left.+\operatorname{Im}\left(C_{1} C_{2}\right) \sin (\Delta \cdot T+\phi / 2)\right] \\
\mathcal{C}= & 4\left|C_{1}\right|^{2}\left|C_{2}\right|^{2}\left|S_{1}\right|^{2}+2\left|C_{1}\right|^{4}\left|S_{2}\right|^{2}+2\left|S_{1}\right|^{4}\left|S_{2}\right|^{2} \\
& +4\left|S_{1}\right|^{2}\left[\operatorname{Re}\left(C_{1}^{2} C_{2}^{2}\right) \cos (\Delta \cdot T+\phi)\right. \\
& \left.+\operatorname{Im}\left(C_{1}^{2} C_{2}^{2}\right) \sin (\Delta \cdot T+\phi)\right]
\end{aligned}
$$

The output has sinusoidal components in $\tau$ with velocity-dependent frequency $\Delta$, and amplitude $\mathcal{A}\left(\Omega, \Delta_{\text {ac }}\right)$, purely constructed from elements of the pulse matrices and thus only dependent on the offset from the light-shifted resonance (with the overall scale determined by the on-resonance Rabi frequency). There is no detuning-dependent phase shift to these harmonic components, though parasitic subharmonics of amplitude $\mathcal{B}\left(\Omega, \Delta, \Delta_{\mathrm{ac}}, \phi, T\right)$ become significant at large detunings. 


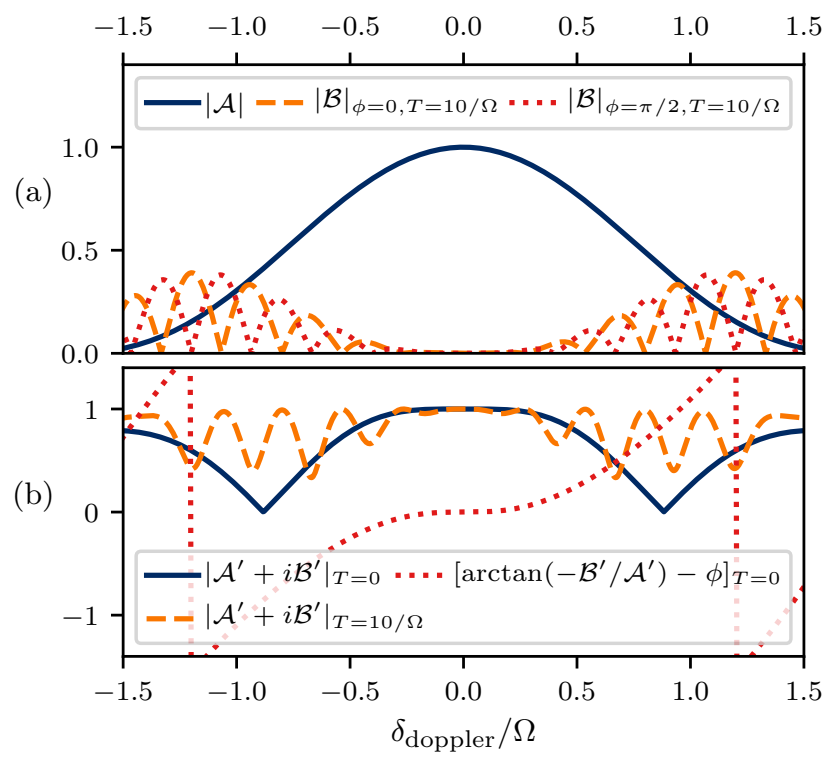

FIG. 8. Amplitudes of frequency components in interferometer outputs as a function of Doppler detuning, in units of the on-resonance Rabi frequency. In these plots $\delta_{\mathrm{ac}}=\delta_{\text {laser }}$; when this is not the case, the profile is shifted in position by the difference. (a) Amplitudes for $T_{1}=T-\tau / 2, T_{2}=T+\tau / 2$ interferometer. Solid (blue) line shows amplitude of $\delta_{\text {doppler }}$ harmonics. Dashed (orange) and dotted (red) lines show the amplitude of $\delta_{\text {doppler }} / 2$ subharmonics with $\phi=0$ and $\phi=\pi / 2$ respectively, when $T=10 / \Omega$. (b) Amplitudes for $T_{1}=T$, $T_{2}=T+\tau$ interferometer. No subharmonics are present, but the $\delta_{\text {doppler }}$ harmonics receive a residual detuning-dependent phase shift, plotted as a dotted (red) line for $T=0$. Solid (blue) line shows the amplitude profile when $T=0$. Dashed (orange) line shows the profile for $T=10 / \Omega$, demonstrating the appearance of an amplitude modulation in lieu of the presence of subharmonics.

With $\mathcal{A}=\mathcal{C}=1, \mathcal{B}=0$, and $\delta_{\text {laser }}=0$, Equation $(\mathrm{A} 8)$ resembles the analogous Ramsey output in the integrand of Equation (4), albeit with an inversion arising from the additional rotation by $\pi$. As long as $\mathcal{A} \gg \mathcal{B}$ then, as in Equation (4), the atomic velocity distribution is well mapped onto the frequency domain and the scaling by $\mathcal{A}$ can be corrected for by multiplying through by its reciprocal.

The magnitudes of $\mathcal{A}$ and $\mathcal{B}$ are plotted as functions of $\delta_{\text {doppler }} / \Omega$ in Figure 8 a which shows that this criterion is satisfied for $-0.4<\delta_{\text {doppler }} / \Omega<0.4$, where $|\mathcal{B} / \mathcal{A}|<$ 0.1. Doppler profiles falling within this window will thus incur little distortion from subharmonics, which would act to artificially narrow broader distributions. As the interferometer time is made longer by increasing $T$, the oscillations in $\mathcal{B}$ become more rapid but remain within the same envelope.

It should be noted that the velocity distribution will be centered about $\delta_{\text {laser }}$ in the frequency domain, so the ambiguity of the $\mathrm{DC}$ component $\mathcal{C}\left(\Omega, \Delta, \Delta_{\mathrm{ac}}, \phi, T\right)$ can be negated by setting $\delta_{\text {laser }}$ much larger than the width

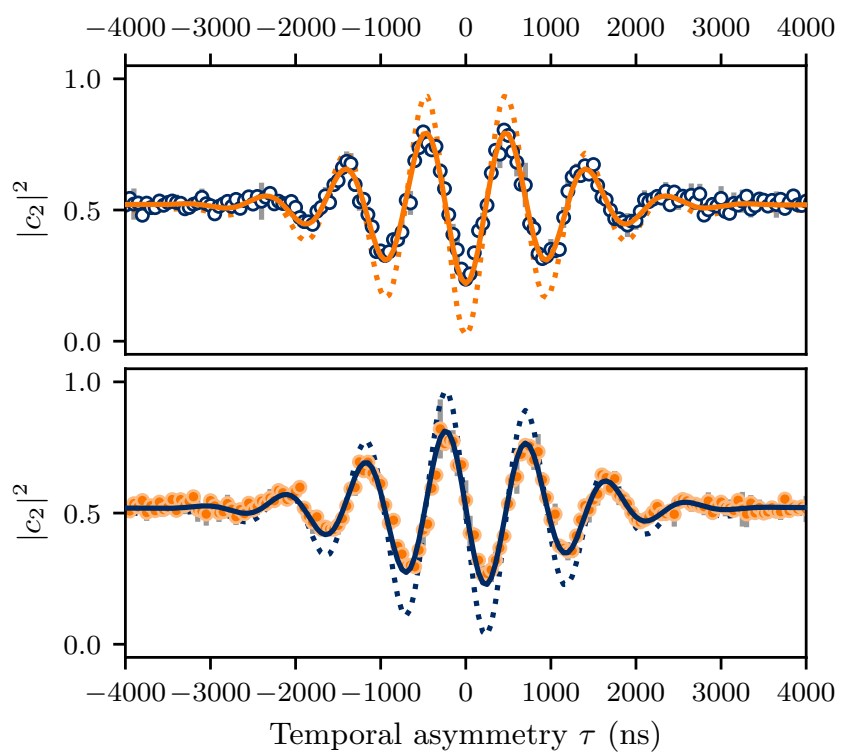

FIG. 9. Empty (blue) and filled (orange) circles respectively show the in-phase and quadrature data from Figure 3. Dashed lines show the output from Equation (A8) integrated over a $33 \mu \mathrm{K}$ velocity distribution, as per Figure 5, with $\delta_{\mathrm{ac}}=-2 \pi \times 1010 \mathrm{kHz}, \delta_{\text {laser }}=-2 \pi \times 1050 \mathrm{kHz}$, and $\Omega=2 \pi \times 685 \mathrm{kHz}$ taken from experimental parameters. Solid lines are the same model vertically centered on the experimental data and scaled by a factor of 0.63 . The contrast loss is expected, as the model does not account for sources of dephasing and finite interaction region present in the real experiment, but the shape otherwise agrees well. We attribute the slight vertical offset in the data to incoherent excitation or imperfect state preparation.

of the Doppler profile and subtracting it off in analysis.

Equation (A8) can be evaluated as a function of $\tau$ and integrated over a distribution of velocities (and hence detunings $\Delta$ ) to predict the interferometer output for the given velocity distribution. This is shown by the dotted lines in Figure 9, which are calculated from the distribution measured in section $\mathrm{V}$ with the same experimental parameters at $\phi=0$ and $\phi=\pi / 2$.

This analysis assumes completely coherent evolution, no phase or amplitude noise on the laser, and no additional phase terms from external fields. Further, it assumes a perfect 2-level atom, as opposed to the ${ }^{85} \mathrm{Rb}$ Raman system with different coupling strengths for magnetic sublevels. Empirically we observe that these factors result in a loss of contrast but do not noticeably affect the shape of the signal, as demonstrated by the solid lines in Figure 9 which have been vertically centered on the experimental data from Figure 3 and scaled by a factor of 0.63 , demonstrating good agreement. We attribute the slight positive offset of the experimental data to imperfect state preparation or incoherent (single-photon) excitation.

Let us now treat case (b), introduced at the beginning 
of this section as the interferometer in which $T_{1}=T$ is kept constant and $T_{2}=T+\tau$, in a similar manner. By expanding the trigonometric functions of Equation (A6), we can isolate the $\tau$ dependence into terms of $\sin (\Delta \tau+\phi)$ and $\cos (\Delta \tau+\phi)$ such that the interferometer output can be written in the form

$$
\left|c_{2}\right|^{2}=\frac{1}{2}\left\{\mathcal{C}^{\prime}+\mathcal{A}^{\prime} \cos (\Delta \tau+\phi)+\mathcal{B}^{\prime} \sin (\Delta \tau+\phi)\right\}
$$

with

$$
\begin{aligned}
& \mathcal{A}^{\prime}=-4\left|C_{1}\right|^{2}\left|S_{2}\right|^{2}\left|S_{1}\right|^{2} \\
&+4\left|S_{1}\right|^{2}\left[\operatorname{Im}\left(C_{1}^{2} C_{2}^{2}\right)-\left|S_{1}\right|\left|S_{2}\right| \operatorname{Im}\left(C_{1} C_{2}\right)\right] \sin (\Delta \cdot T) \\
&+4\left|S_{1}\right|^{2}\left[\operatorname{Re}\left(C_{1}^{2} C_{2}^{2}\right)-\left|S_{1}\right|\left|S_{2}\right| \operatorname{Re}\left(C_{1} C_{2}\right)\right] \cos (\Delta \cdot T) \\
&+4\left|C_{1}\right|^{2}\left|S_{1}\right|\left|S_{2}\right|\left[\operatorname{Im}\left(C_{1} C_{2}\right) \sin (\Delta \cdot T)\right. \\
&\left.+\operatorname{Re}\left(C_{1} C_{2}\right) \cos (\Delta \cdot T)\right], \quad \text { (A11a) } \\
& \mathcal{B}^{\prime}= 4\left[\left|S_{1}\right|^{2} \operatorname{Im}\left(C_{1}^{2} C_{2}^{2}\right)+\left(\left|C_{1}\right|^{2}-\left|S_{1}\right|^{2}\right)\right. \\
&\left.\times\left|S_{1}\right|\left|S_{2}\right| \operatorname{Im}\left(C_{1} C_{2}\right)\right] \cos (\Delta \cdot T) \\
&-4\left[\left|S_{1}\right|^{2} \operatorname{Re}\left(C_{1}^{2} C_{2}^{2}\right)+\left(\left|C_{1}\right|^{2}-\left|S_{1}\right|^{2}\right)\right. \\
&\left.\times\left|S_{1}\right|\left|S_{2}\right| \operatorname{Re}\left(C_{1} C_{2}\right)\right] \sin (\Delta \cdot T), \\
& \mathcal{C}^{\prime}=2\left|C_{1}\right|^{4}\left|S_{2}\right|^{2}+2\left|S_{1}\right|^{4}\left|S_{2}\right|^{2}+4\left|C_{1}\right|^{2}\left|C_{2}\right|^{2}\left|S_{1}\right|^{2} \\
&-4\left|S_{1}\right|^{3}\left|S_{2}\right|\left[\operatorname{Re}\left(C_{1} C_{2}\right) \cos (\Delta \cdot T)\right. \\
&\left.+\operatorname{Im}\left(C_{1} C_{2}\right) \sin (\Delta \cdot T)\right] \\
&+4\left|C_{1}\right|^{2}\left|S_{1}\right|\left|S_{2}\right|\left[\operatorname{Re}\left(C_{1} C_{2}\right) \cos (\Delta \cdot T)\right. \\
&\left.+\operatorname{Im}\left(C_{1} C_{2}\right) \sin (\Delta \cdot T)\right] .
\end{aligned}
$$

Subharmonic components are no longer present in this output, but quadrature terms are, introducing an effective detuning-dependent phase shift $\arctan \left(-\mathcal{B}^{\prime} / \mathcal{A}^{\prime}\right)$ which, unlike the Ramsey interferometer [27], has a flat gradient through $\Delta_{\mathrm{ac}}=0 .\left|\mathcal{A}^{\prime}+i \mathcal{B}^{\prime}\right|$ gives the amplitude of the harmonic components which, for $T>0$, exhibits an oscillatory modulation, the envelope of which resembles that of the subharmonics present in case (a).

The case of $T=0$ warrants special attention. This reduces to a 2 -pulse $3 \pi / 2-\pi / 2$ interferometer, restricting the pulse separation to $\tau>0$. This can still yield good velocimetry results when the gradient of the detuningdependent phase shift is small, as it is about $\Delta_{\mathrm{ac}}=0$, unlike in a Ramsey interferometer [27]. This case is considered in Appendix B.

\section{Appendix B: Enhanced Ramsey interferometer}

In a two-pulse interferometer, data collection is inherently restricted to the $\tau>0$ window, mathematically expressed by multiplying the time domain output by the Heaviside step function $\Theta(\tau)$. In the frequency (velocity)

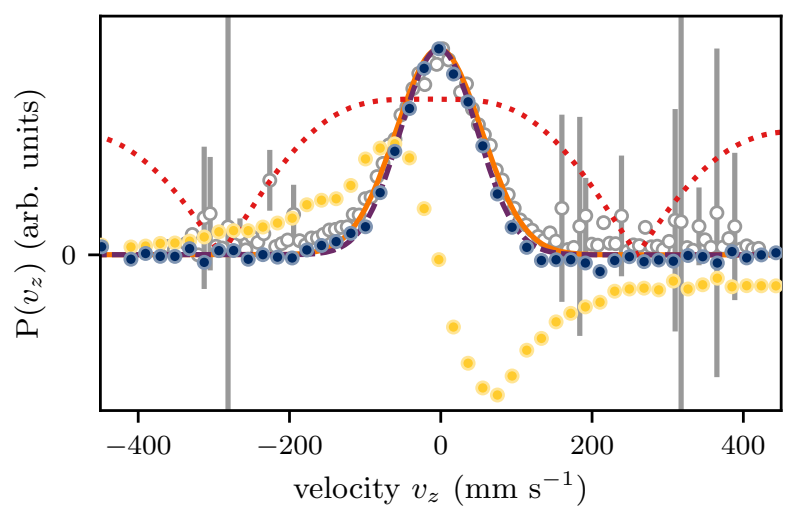

FIG. 10. Interferometric velocimetry measurement with enhanced Ramsey interferometer. Real and imaginary parts of the FFT are shown by dark (blue) and light (yellow) filled circles respectively, overlaid upon a Doppler spectroscopy profile, empty circles (gray), taken under the same conditions. The real part of the interferometric measurement shows good agreement with the spectroscopic one. The solid (orange) and dashed (purple) lines show Gaussian fits to the spectroscopic and interferometric data respectively, with corresponding temperatures of $26 \mu \mathrm{K}$ and $33 \mu \mathrm{K}$. The theoretical amplitude $\left|\mathcal{A}^{\prime}+i \mathcal{B}^{\prime}\right|$ from Figure $8 \mathrm{~b}$ is shown as an additional dashed (red) line, plotted from measured experimental parameters.

domain, this manifests as a convolution with the Fourier transform of the step function

$$
\tilde{\Theta}(\omega)=\frac{i}{\sqrt{2 \pi} \omega}+\sqrt{\frac{\pi}{2}} \delta(\omega)
$$

$\delta(\omega)$ being the Dirac delta function. This introduced an imaginary component to the output, but leaves the velocity distribution unaltered in the real part.

In a realistic Ramsey interferometer (Figure 1b) where the pulses are of finite length, setting the separation to $\tau=0$ is not equivalent to setting the effective interferometer period to 0 . The result of this is a multiplication by $\Theta(\tau-\delta \tau)$, where $\delta \tau$ is an offset determined by the length of the pulses. This introduces a phase factor into the first term of Equation (B1), irreversibly mixing the real part of the $\delta \tau=0$ output with the imaginary part so that the velocity distribution is irretrievable from a single measurement [27].

As touched upon at the end of Appendix A, the asymmetric Mach-Zehnder interferometer case (b) with $T=0$ is a two-pulse $3 \pi / 2-\pi / 2$ interferometer. This has the properties of a Ramsey-type interferometer, but with a degree of cancellation of the phase picked up during the pulses. While the Ramsey interferometer has a linear dependence of the phase shift on detuning, equivalent to an offset of the effective time origin, for the $3 \pi / 2-\pi / 2$ interferometer the lowest order term in the phase shift is cubic. This gives it a flat gradient about zero detuning, a property shared by the shift in amplitude (Figure 8b). 
Preliminary results, shown in Figure 10, show that the real parts of the Fourier transformed interferometer provide an effective measure of the velocity profile. Distortion due to the time domain truncation is primarily restricted to the imaginary parts. The detuning-dependent amplitude from Figure 8b, plotted from measured experimental parameters, is shown as a red (dotted) line; though the data have not been corrected by multiplying by its reciprocal. The reduction in amplitude is $<10 \%$ across 2 standard deviations (95\%) of the Gaussian distribution fitted to the real data, which are negligibly affected by correction (although the imaginary data are distorted). This broad, flat, profile offers a potential advantage of what we term the enhanced Ramsey method.

The measurement is overlaid upon a spectroscopic measurement taken under the same conditions and they are is good agreement, with the interferometric measurement appearing slightly narrower. We can attribute this discrepancy to artefacts of the Doppler spectroscopy discussed in Section VI and sensitivity of the interferometric measurement to pulse rise-time at small $\tau$.

\section{ACKNOWLEDGMENTS}

This work was supported by the EPSRC through the UK Quantum Technology Hub for Sensors \& Metrology under grant EP/M013294/1, and by Dstl under contracts DSTLX-1000091758 and DSTLX-1000097855. The data presented in this paper are available for download from http://doi.org/10.5258/SOTON/D0383.
[1] M. Hohmann, F. Kindermann, T. Lausch, D. Mayer, F. Schmidt, and A. Widera, Physical Review A 93, 043607 (2016).

[2] J. Millen and A. Xuereb, New Journal of Physics 18, 011002 (2016).

[3] P. D. Lett, R. N. Watts, C. I. Westbrook, W. D. Phillips, P. L. Gould, and H. J. Metcalf, Physical Review Letters 61, 169 (1988).

[4] M. Kasevich, D. S. Weiss, E. Riis, K. Moler, S. Kasapi, and S. Chu, Physical Review Letters 66, 2297 (1991).

[5] I. G. Hughes, Journal of Modern Optics 65, 640 (2018).

[6] J. Stenger, S. Inouye, A. P. Chikkatur, D. M. StamperKurn, D. E. Pritchard, and W. Ketterle, Physical Review Letters 82, 4569 (1999).

[7] B. Deh, C. Marzok, S. Slama, C. Zimmermann, and P. W. Courteille, Applied Physics B 97, 387 (2009).

[8] D.-S. Lü, Q.-Z. Qu, B. Wang, J.-B. Zhao, L. Liu, and Y.-Z. Wang, Chinese Physics Letters 28, 063201 (2011).

[9] P. Zhang, Y. Guo, Z. Li, Y.-c. Zhang, Y. Zhang, J. Du, G. Li, J. Wang, and T. Zhang, Journal of the Optical Society of America B 28, 667 (2011).

[10] S. Pradhan and B. N. Jagatap, Review of Scientific Instruments 79, 013101 (2008).

[11] T. M. Brzozowski, M. Maczynska, M. Zawada, J. Zachorowski, and W. Gawlik, Journal of Optics B: Quantum and Semiclassical Optics 4, 62 (2002).

[12] P. D. Featonby, G. S. Summy, C. L. Webb, R. M. Godun, M. K. Oberthaler, A. C. Wilson, C. J. Foot, and K. Burnett, Physical Review Letters 81, 495 (1998).

[13] B. Saubaméa, T. W. Hijmans, S. Kulin, E. Rasel, E. Peik, M. Leduc, and C. Cohen-Tannoudji, Physical Review Letters 79, 3146 (1997).

[14] E. Maréchal, R. Long, T. Miossec, J.-L. Bossennec, R. Barbé, J.-C. Keller, and O. Gorceix, Physical Review A 62, 053603 (2000).

[15] S. Riedl, G. W. Hoth, B. Pelle, J. Kitching, and E. A. Donley, Journal of Physics: Conference Series 723, 012058 (2016).

[16] A. Peters, K. Y. Chung, and S. Chu, Metrologia 38, 25 (2001).

[17] T. L. Gustavson, A. Landragin, and M. A. Kasevich, Classical and Quantum Gravity 17, 2385 (2000).
[18] M. Kasevich and S. Chu, Physical Review Letters 67, 181 (1991).

[19] B. Barrett, A. Bertoldi, and P. Bouyer, Physica Scripta 91, 053006 (2016).

[20] G. D'Amico, L. Cacciapuoti, M. Jain, S. Zhan, and G. Rosi, (2018), arXiv:1803.11492.

[21] C. J. Bordé, Physics Letters A 140, 10 (1989).

[22] N. F. Ramsey, Physical Review 78, 695 (1950).

[23] K. Bongs, R. Launay, and M. Kasevich, Applied Physics B 84, 599 (2006).

[24] P. R. Berman, Atom Interferometry (Elsevier Science, 1996).

[25] A. A. Michelson, The London, Edinburgh, and Dublin Philosophical Magazine and Journal of Science 31, 338 (1891).

[26] R. Bell, Introductory Fourier transform spectroscopy (Academic Press, 1972).

[27] M. Carey, M. Belal, M. Himsworth, J. Bateman, and T. Freegarde, Journal of Modern Optics 65, 657 (2018).

[28] In practise the time series data is unavoidably truncated, and the time origin determines how the velocity distribution is divided between the real and imaginary parts of the transform, but the absolute value always offers a good measure.

[29] A. Dunning, R. Gregory, J. Bateman, N. Cooper, M. Himsworth, J. A. Jones, and T. Freegarde, Physical Review A 90, 033608 (2014).

[30] P. Gillot, B. Cheng, S. Merlet, and F. Pereira Dos Santos, Physical Review A 93, 013609 (2016).

[31] A. Dunning, R. Gregory, J. Bateman, M. Himsworth, and T. Freegarde, Physical Review Letters 115, 073004 (2015).

[32] The stochastic optical pumping process from the $\left|5 S_{1 / 2}, F=3\right\rangle$ to $\left|5 S_{1 / 2}, F=2\right\rangle$ state via the MOT cooling beam has a $1 / e$ time constant on the order of $100 \mu \mathrm{s}$, depending on the detuning and power of the cooling beam that are tweaked to control the atomic temperature. The transfer from the $\left|5 S_{1 / 2}, F=2\right\rangle$ to $\left|5 S_{1 / 2}, F=3\right\rangle$ state via the resonant repump beam is faster by an order of magnitude. Imperfect state preparation causes a loss of fringe visibility without affecting the form of the signal.

[33] N. Cooper, J. Woods, J. Bateman, A. Dunning, and 
T. Freegarde, Applied Optics 52, 5713 (2013).

[34] A. Dunning, Coherent atomic manipulation and cooling using composite optical pulse sequences, Ph.D. thesis, University of Southampton (2014), https://eprints . soton.ac.uk/364735. Also published by Springer, DOI:10.1007/978-3-319-21738-3.

[35] C. G. Townsend, N. H. Edwards, C. J. Cooper, K. P. Zetie, C. J. Foot, A. M. Steane, P. Szriftgiser, H. Perrin, and J. Dalibard, Physical Review A 52, 1423 (1995).

[36] L. P. Parazzoli, A. M. Hankin, and G. W. Biedermann, Physical Review Letters 109, 230401 (2012).

[37] M. H. Anderson, J. R. Ensher, M. R. Matthews, C. E.
Wieman, and E. A. Cornell, Science (New York, N.Y.) 269, 198 (1995).

[38] G. Afek, J. Coslovsky, A. Courvoisier, O. Livneh, and N. Davidson, Physical Review Letters 119, 060602 (2017).

[39] I. I. Rabi, N. F. Ramsey, and J. Schwinger, Reviews of Modern Physics 26, 167 (1954).

[40] R. P. Feynman, F. L. Vernon, and R. W. Hellwarth, Journal of Applied Physics 28, 49 (1957).

[41] R. Stoner, D. Butts, J. Kinast, and B. Timmons, Journal of the Optical Society of America B 28, 2418 (2011). 